PROCEEDINGS OF THE

AMERICAN MATHEMATICAL SOCIETY

Volume 130, Number 3, Pages 779-789

S 0002-9939(01)06111-1

Article electronically published on August 29, 2001

\title{
LARGE TIME BEHAVIOR OF SMALL SOLUTIONS TO SUBCRITICAL DERIVATIVE NONLINEAR SCHRÖDINGER EQUATIONS
}

\author{
NAKAO HAYASHI, PAVEL I. NAUMKIN, AND YASUKO YAMAZAKI
}

(Communicated by Christopher D. Sogge)

AbStract. We consider the derivative nonlinear Schrödinger equations

$$
\left\{\begin{array}{c}
i u_{t}+\frac{1}{2} u_{x x}=a(t) F\left(u, u_{x}\right), \quad(t, x) \in \mathbf{R}^{2}, \\
u(0, x)=\epsilon u_{0}(x), \quad x \in \mathbf{R},
\end{array}\right.
$$

where the coefficient $a(t)$ satisfies the time growth condition

$$
|a(t)| \leq C(1+|t|)^{1-\delta}, \quad 0<\delta<1
$$

$\epsilon$ is a sufficiently small constant and the nonlinear interaction term $F$ consists of cubic nonlinearities of derivative type

$$
\begin{aligned}
F\left(u, u_{x}\right)= & \lambda_{1}|u|^{2} u+i \lambda_{2}|u|^{2} u_{x}+i \lambda_{3} u^{2} \bar{u}_{x} \\
& +\lambda_{4}\left|u_{x}\right|^{2} u+\lambda_{5} \bar{u} u_{x}^{2}+i \lambda_{6}\left|u_{x}\right|^{2} u_{x},
\end{aligned}
$$

where $\lambda_{1}, \lambda_{6} \in \mathbf{R}, \lambda_{2}, \lambda_{3}, \lambda_{4}, \lambda_{5} \in \mathbf{C}, \lambda_{2}-\lambda_{3} \in \mathbf{R}$, and $\lambda_{4}-\lambda_{5} \in \mathbf{R}$. We suppose that the initial data satifsfy the exponential decay conditions. Then we prove the sharp decay estimate $\|u(t)\|_{\mathbf{L}^{p}} \leq C \epsilon t^{\frac{1}{p}-\frac{1}{2}}$, for all $t \geq 1$, where $2 \leq p \leq \infty$. Furthermore we show that for $\frac{1}{2}<\delta<1$ there exist the usual scattering states, when $b(x)=\lambda_{1}-\left(\lambda_{2}-\lambda_{3}\right) x+\left(\lambda_{4}-\lambda_{5}\right) x^{2}-\lambda_{6} x^{3}=0$, and the modified scattering states, when $b(x) \neq 0$.

\section{INTRODUCTION}

We consider the Cauchy problem for the cubic derivative nonlinear Schrödinger equation

$$
\left\{\begin{array}{c}
i u_{t}+\frac{1}{2} u_{x x}=a(t) F\left(u, u_{x}\right), \quad(t, x) \in \mathbf{R}^{2}, \\
u(0, x)=\epsilon u_{0}(x), \quad x \in \mathbf{R}
\end{array}\right.
$$

where the coefficient $a(t)$ satisfies the time growth condition $|a(t)| \leq C(1+|t|)^{1-\delta}$, $0<\delta<1, \epsilon$ is a sufficiently small constant and the nonlinear interaction term $F$ consists of the cubic nonlinearities of derivative type

$$
\begin{aligned}
F\left(u, u_{x}\right)= & \lambda_{1}|u|^{2} u+i \lambda_{2}|u|^{2} u_{x}+i \lambda_{3} u^{2} \bar{u}_{x}+\lambda_{4}\left|u_{x}\right|^{2} u \\
& +\lambda_{5} \bar{u} u_{x}^{2}+i \lambda_{6}\left|u_{x}\right|^{2} u_{x}
\end{aligned}
$$

Received by the editors May 22, 2000 and, in revised form, September 15, 2000.

2000 Mathematics Subject Classification. Primary 35Q55.

Key words and phrases. Subcritical nonlinear Schrödinger equations, large time asymptotics, scattering problem. 
where the coefficients $\lambda_{1}, \lambda_{6} \in \mathbf{R}, \lambda_{2}, \lambda_{3}, \lambda_{4}, \lambda_{5} \in \mathbf{C}, \lambda_{2}-\lambda_{3} \in \mathbf{R}$, and $\lambda_{4}-\lambda_{5} \in \mathbf{R}$. We propose to classify the nonlinearities in a nonlinear Schrödinger equation as follows. If the usual scattering states exist in $\mathbf{L}^{2}$, we call the nonlinearity super critical. If the usual scattering states do not exist and the $\mathbf{L}^{2}$ norm of the nonlinear term decays like $O\left(t^{-\delta}\right)$, we call it critical if $\delta=1$, and subcritical if $\delta<1$. Our main result in the present paper says that nonlinearity (1.2) is super critical if $b(\xi)=0$ and $1 / 2<\delta \leq 1$, where

$$
b(\xi)=\lambda_{1}-\left(\lambda_{2}-\lambda_{3}\right) \xi+\left(\lambda_{4}-\lambda_{5}\right) \xi^{2}-\lambda_{6} \xi^{3},
$$

it is critical if $b(\xi) \neq 0$ and $\delta=1$, and it is subcritical if $b(\xi) \neq 0$ and $\delta \in(0,1)$. In the case of power nonlinearity $|u|^{p-1} u$, it appears to be super critical for $p>3$, critical for $p=3$, and subcritical for $1<p<3$. A nonlocal nonlinear Schrödinger equation with nonlinearity $\int|x-y|^{-\delta}|u|^{2} d y u, \delta \in(0,1)$, is also classified as subcritical (see paper [12]). Note that a cubic nonlinearity is not necessarily critical; for example, the nonlinearity $\partial_{x}\left(u^{3}\right)$ is super critical due to its oscillating structure (see [13]). Also note that the quadratic nonlinearity $\left(u_{x}\right)^{2}$ is super critical (see [22]) and we do not know when a quadratic nonlinearity is critical or subcritical. There are many works concerning the nonlinear Schrödinger equations with power nonlinearities. In the super critical case the scattering problem was studied in papers [3], [4], [8], [17], [19], [23], [24], [25] and in the critical case it was treated in [2], [11], [15], 20], [21. The existence of modified scattering states was shown in papers [11, [15], [20] and modified wave operators were constructed in 2], 21]. In our previous paper [15] we considered the Cauchy problem (1.1) with cubic derivative nonlinearity (1.2), when the coefficient $\delta=1$. We proved that the solution of (1.1) with (1.2) and $\delta=1$ exists and satisfies the sharp time decay estimate $\|u(t)\|_{\mathbf{L}^{p}} \leq C \epsilon t^{1 / p-1 / 2}$ for all $t \geq 1$, where $2 \leq p \leq \infty$, if the initial function $u_{0}$ is in the weighted Sobolev space $\mathbf{H}^{3,0} \cap \mathbf{H}^{2,1}$, where $\mathbf{H}^{m, s}=\left\{\phi \in \mathbf{L}^{2} ;\left\|\langle x\rangle^{s}\left\langle i \partial_{x}\right\rangle^{m} \phi\right\|_{\mathbf{L}^{2}}<\infty\right\},\langle x\rangle=\sqrt{1+x^{2}}$. Moreover we showed that there exists a unique final state $u_{+} \in \mathbf{L}^{2}$ such that

$$
\left\|u(t)-\exp \left(-i\left|\hat{u}_{+}(\cdot / t)\right|^{2} b(\cdot / t) \log t\right) \mathcal{U}(t) u_{+}\right\|_{\mathbf{L}^{2}}=O\left(t^{-1 / 2-\alpha}\right)
$$

for all $t \geq 1$, where $0<\alpha<1 / 4, \hat{\phi}$ denotes the Fourier transform of $\phi$, and $\mathcal{U}(t)=e^{-i t \partial_{x}^{2} / 2}$ is the free Schrödinger evolution group. The aim of the present work is to prove the results of paper [15] for the more difficult subcritical cases. As far as we know there are no results for the scattering problem in subcritical cases except the case of the nonlinear Schrödinger equation without derivatives of unknown function in the nonlinear term (see [5], 6], 7], [9], [10, [12]). The main point in the proof of our results is the application of the transformation for the solution $u(t)=\mathcal{M}(t) \mathcal{D}(t) v(t)$ (see [16]), where $\mathcal{M}=\mathcal{M}(t)=\exp \left(i x^{2} / 2 t\right.$ ) and $\mathcal{D}(t)$ is the dilation operator defined by $(\mathcal{D}(t) \psi)(x)=(1 / \sqrt{i t}) \psi(x / t)$. The idea of the transformation can be understood from the large time asymptotic expansion of the solution of the linear Schrödinger equation. But the miracle property of the transformation is that the nonlinearity can also be easily transformed and in the resulting equation for the new unknown function $v(t)$ we can extract the divergent nonlinear terms explicitly. To cancel these divergent terms we represent the solution $v(t)$ in the rapidly oscillating form $v=w e^{i g}$ with slowly varying amplitude $w(t)$ and growing with time phase function $g(t)$. However the system of equations for $w$ and $g$ has the so-called difficulty of the derivative loss. In order to prove the existence of the solution $(w, g)$ we apply the estimates in the analytic function 
space $\mathcal{H}_{\sigma(t)}^{5 / 2,3}$, where $\sigma(t)=\beta\left(1+(1+t)^{-\gamma}\right) / 2, \gamma \in(0, \delta / 2]$. Analytic function spaces were used by many authors to overcome the derivative loss in the nonlinear evolution equations (e.g., see [1], [10], 9], [18]). In what follows we consider the positive time $t$ only, since for the negative one the results are analogous. We use the following notation and function spaces. Let $\mathcal{F} \phi$ or $\hat{\phi}$ be the Fourier transform of $\phi$ defined by $\mathcal{F} \phi(\chi)=(2 \pi)^{-1 / 2} \int e^{-i x \chi} \phi(x) d x$ and $\mathcal{F}^{-1} \phi(x)$ be the inverse Fourier transform of $\phi$, i.e. $\mathcal{F}^{-1} \phi(x)=(2 \pi)^{-1 / 2} \int e^{i x \chi} \phi(\chi) d \chi$. We introduce some function spaces. The usual Lebesgue space is $\mathbf{L}^{p}=\left\{\phi \in \mathbf{S}^{\prime} ;\|\phi\|_{p}<\infty\right\}$ where the norm $\|\phi\|_{p}=\left(\int|\phi(x)|^{p} d x\right)^{1 / p}$ if $1 \leq p<\infty$ and $\|\phi\|_{\infty}=\operatorname{ess.sup}\{|\phi(x)| ; x \in \mathbf{R}\}$ if $p=$ $\infty$. For simplicity we denote $\|\phi\|=\|\phi\|_{2}$. The weighted Sobolev space is $\mathbf{H}^{m, s}=$ $\left\{\phi \in \mathbf{S}^{\prime} ;\left\|\langle x\rangle^{s}\left\langle i \partial_{x}\right\rangle^{m} \phi\right\|<\infty\right\}, m, s \in \mathbf{R}$, with norm $\|\phi\|_{m, s}=\left\|\langle x\rangle^{s}\left\langle i \partial_{x}\right\rangle^{m} \phi\right\|$ and the homogeneous Sobolev space is $\dot{\mathbf{H}}^{m, s}=\left\{\phi \in \mathbf{S}^{\prime} ;\left\||x|^{s}\left(-\partial_{x}^{2}\right)^{m / 2} \phi\right\|<\infty\right\}$ with seminorm $\|\phi\|_{\dot{\mathbf{H}}^{m, s}}=\left\||x|^{s}\left(-\partial_{x}^{2}\right)^{m / 2} \phi\right\|$. Also we define the analytic function space $\mathcal{H}_{\sigma}^{m, s}=\left\{\phi \in \mathbf{L}^{2} ;\left\|e^{\sigma|p|}\langle p\rangle^{m}\left\langle i \partial_{p}\right\rangle^{s} \hat{\phi}(p)\right\|<\infty\right\}, m, s \in \mathbf{R}$, with norm $\|\phi\|_{\mathcal{H}_{\sigma}^{m, s}}=$ $\left\|e^{\sigma|p|}\langle p\rangle^{m}\left\langle i \partial_{p}\right\rangle^{s} \hat{\phi}(p)\right\|$, which can be expressed in $x$-representation in terms of the analyticity in the strip $-\sigma \leq \Im z \leq \sigma$ via the following norm: $\|\phi(\cdot+i \sigma)\|_{\mathbf{H}^{m, s}}+$ $\|\phi(\cdot-i \sigma)\|_{\mathbf{H}^{m, s}}$. Indeed we have the inequalities $\frac{1}{C}\|\phi\|_{\mathcal{H}_{\sigma}^{m, s}} \leq\|\phi(\cdot+i \sigma)\|_{\mathbf{H}^{m, s}}+$ $\|\phi(\cdot-i \sigma)\|_{\mathbf{H}^{m, s}} \leq C\|\phi\|_{\mathcal{H}_{\sigma}^{m, s}}$ with $C>0$. We denote the inner product in $\mathbf{L}^{2}$ by $(\psi, \varphi)=\int \psi(x) \cdot \bar{\varphi}(x) d x$. The free Schrödinger evolution group $\mathcal{U}(t)$ gives us the solution of the linear Cauchy problem (1.1) (with $F=0$ ). It can be represented explicitly in the following manner:

$$
\mathcal{U}(t) \phi=(2 \pi i t)^{-1 / 2} \int e^{i(x-y)^{2} / 2 t} \phi(y) d y=\mathcal{F}^{-1} e^{i t \chi^{2} / 2} \mathcal{F} \phi .
$$

Note that $\mathcal{U}(t)=\mathcal{M}(t) \mathcal{D}(t) \mathcal{F} \mathcal{M}(t)$. Then since $\mathcal{D}^{-1}(t)=i \mathcal{D}(1 / t)$ we have $\mathcal{U}(-t)=\overline{\mathcal{M}} \mathcal{F}^{-1} \mathcal{D}^{-1}(t) \overline{\mathcal{M}}=i \overline{\mathcal{M}} \mathcal{F}^{-1} \mathcal{D}(1 / t) \overline{\mathcal{M}}$, where $\overline{\mathcal{M}}=\mathcal{M}(-t)$. By $\mathbf{C}(\mathbf{I} ; \mathbf{B})$ we denote the space of continuous functions from an interval $\mathbf{I}$ to a Banach space B. Different positive constants might be denoted by the same letter $C$.

We now state our results.

Theorem 1.1. We assume that $e^{\beta\langle x\rangle} u_{0} \in \mathbf{H}^{3,5 / 2}, \beta>0$. Then there exists a unique global solution of the Cauchy problem for the cubic derivative nonlinear Schrödinger equation (1.1) such that $u \in \mathbf{C}\left([0, \infty) ; \mathbf{H}^{3,0}\right), \mathcal{F U}(-t) u(t) \in$ $\mathbf{C}\left([0, \infty) ; \mathcal{H}_{\beta / 2}^{5 / 2,3}\right)$, and the decay estimate

$$
\|u(t)\|_{p} \leq C \varepsilon t^{1 / p-1 / 2}
$$

is valid for all $t \geq 1$, where $2 \leq p \leq \infty$. Moreover there exists a unique final state $\hat{u}_{+} \in \mathcal{H}_{\beta / 2}^{0,3}$ such that the asymptotics

$$
\begin{aligned}
u(t, x)= & (i t)^{-1 / 2} \hat{u}_{+}(x / t) \exp \left(i x^{2} / 2 t-i \int_{0}^{t} a(\tau) /(\tau+1) d \tau b(x / t)\left|\hat{u}_{+}(x / t)\right|^{2}\right. \\
& \left.+O\left(1+t^{1-2 \delta}\right)\right)+O\left(t^{-1 / 2-\delta}\right)
\end{aligned}
$$

is true for $t \rightarrow \infty$ uniformly with respect to $x \in \mathbf{R}$, where $b$ is defined in (1.3).

For the values $\delta \in(1 / 2,1)$ we obtain the existence of the modified scattering states. 
Theorem 1.2. Let $u$ be the solution of the Cauchy problem (1.1) obtained in Theorem 1.1 and $1 / 2<\delta<1$. Then there exists a unique final state $\hat{u}_{+} \in \mathcal{H}_{\beta / 2}^{0,3}$ such that the asymptotics

$$
\begin{aligned}
u(t, x)= & (i t)^{-1 / 2} \hat{u}_{+}(x / t) \exp \left(i x^{2} / 2 t-i \int_{0}^{t} a(\tau) /(\tau+1) d \tau b(x / t)\left|\hat{u}_{+}(x / t)\right|^{2}\right) \\
& +O\left(t^{-1 / 2-\delta}\right)
\end{aligned}
$$

is valid for $t \rightarrow \infty$ uniformly with respect to $x \in \mathbf{R}$ and the estimate

$$
\left\|u(t)-\exp \left(-i \int_{0}^{t} a(\tau) /(\tau+1) d \tau b(\cdot / t)\left|\hat{u}_{+}(\cdot / t)\right|^{2}\right) \mathcal{U}(t) u_{+}\right\| \leq C t^{1-2 \delta}
$$

is true for all $t \geq 1$.

Remark 1.1. The results similar to that of Theorems 1.1-1.2 in the particular case $\lambda_{2}=\lambda_{3}=\lambda_{4}=\lambda_{5}=\lambda_{6}=0$ were obtained in papers [9], [12].

We organize our paper as follows. In Section 2 we derive the system of equations for the amplitude $w$ and the phase $g$. Then in Theorem 2.1 we prove the existence and estimates of the solution $(w, g)$ in the analytic function space $\mathcal{H}_{\sigma(t)}^{5 / 2,3}$. Section 3 is devoted to the proof of Theorems 1.1-1.2.

\section{Preliminary estimates}

We define a new function $v(\tau, \xi)=e^{-i \xi^{2} \tau / 2} \mathcal{D}^{-1}(\tau) u(t)=\mathcal{B}(\tau) u(t)$, where $\xi=$ $x / \tau, \tau=t+1, \mathcal{B}(\tau)=\mathcal{F} \mathcal{M}(\tau) \mathcal{U}(-\tau)$. In the same way as in [16] we see that $v(\tau)$ satisfies the equation $\mathcal{L} v=a(\tau-1) \mathcal{B}(\tau) F\left(u, u_{x}\right)$, where $\mathcal{L}=i \partial_{\tau}+\partial_{\xi}^{2} / 2 \tau^{2}$. By a direct computation we have $\mathcal{B}(\tau) u_{x}=i \xi v+v_{\xi} / \tau$, hence $\mathcal{B}(\tau) F\left(u, u_{x}\right)=F_{1} / \tau$, where

$$
\begin{aligned}
F_{1}= & F\left(v, i \xi v+\frac{1}{\tau} v_{\xi}\right)=b|v|^{2} v+\frac{i \mu_{2}}{\tau}|v|^{2} v_{\xi}+\frac{i \mu_{3}}{\tau} v^{2} \bar{v}_{\xi} \\
& +\frac{\mu_{4}}{\tau^{2}}\left|v_{\xi}\right|^{2} v+\frac{\mu_{5}}{\tau^{2}} \bar{v} v_{\xi}^{2}+\frac{i \lambda_{6}}{\tau^{3}}\left|v_{\xi}\right|^{2} v_{\xi}
\end{aligned}
$$

and $b=\lambda_{1}-\left(\lambda_{2}-\lambda_{3}\right) \xi+\left(\lambda_{4}-\lambda_{5}\right) \xi^{2}-\lambda_{6} \xi^{3}, \mu_{2}=\lambda_{2}-\left(\lambda_{4}-2 \lambda_{5}\right) \xi+2 \lambda_{6} \xi^{2}$, $\mu_{3}=\lambda_{3}+\lambda_{4} \xi-\lambda_{6} \xi^{2}, \mu_{4}=\lambda_{4}-2 \lambda_{6} \xi, \mu_{5}=\lambda_{5}+\lambda_{6} \xi$. Thus we have

$$
\begin{aligned}
\mathcal{L} v=\frac{a}{\tau} & \left(b|v|^{2} v+\frac{i \mu_{2}}{\tau}|v|^{2} v_{\xi}+\frac{i \mu_{3}}{\tau} v^{2} \bar{v}_{\xi}+\frac{\mu_{4}}{\tau^{2}}\left|v_{\xi}\right|^{2} v\right. \\
& \left.+\frac{\mu_{5}}{\tau^{2}} \bar{v} v_{\xi}^{2}+\frac{i \lambda_{6}}{\tau^{3}}\left|v_{\xi}\right|^{2} v_{\xi}\right) .
\end{aligned}
$$

Time decay of the first summand in the right-hand side of equation (2.1) is not sufficient for obtaining the existence of global solutions with sharp decay estimate. In order to remove this term we introduce a phase function, putting $v=w e^{i g}$, where a real-valued function $g$ will be determined later. Then we get

$$
e^{-i g} F_{1}=q|w|^{2} w+Q
$$

where

$$
\begin{gathered}
q=q\left(g_{\xi}\right)=b-\left(\mu_{2}-\mu_{3}\right) g_{\xi} / \tau+\left(\mu_{4}-\mu_{5}\right)\left(g_{\xi} / \tau\right)^{2}-\lambda_{6}\left(g_{\xi} / \tau\right)^{3} \\
Q=Q\left(w, w_{\xi}, g_{\xi}\right)=\frac{i \nu_{2}}{\tau}|w|^{2} w_{\xi}+\frac{i \nu_{3}}{\tau} w^{2} \bar{w}_{\xi}+\frac{\nu_{4}}{\tau^{2}}\left|w_{\xi}\right|^{2} w+\frac{\nu_{5}}{\tau^{2}} \bar{w} w_{\xi}^{2}+\frac{i \lambda_{6}}{\tau^{3}}\left|w_{\xi}\right|^{2} w_{\xi}
\end{gathered}
$$


and the coefficients $\nu_{j}=\nu_{j}\left(g_{\xi}\right), j=2, \ldots, 5$, are defined as follows: $\nu_{2}=\mu_{2}-$ $\left(\mu_{4}-2 \mu_{5}\right) g_{\xi} / \tau+2 \lambda_{6}\left(g_{\xi} / \tau\right)^{2}, \nu_{3}=\mu_{3}+\mu_{4} g_{\xi} / \tau-\lambda_{6}\left(g_{\xi} / \tau\right)^{2}, \nu_{4}=\mu_{4}-2 \lambda_{6} g_{\xi} / \tau$, and $\nu_{5}=\mu_{5}+\lambda_{6} g_{\xi} / \tau$. Multiplying both sides of equation (2.1) by $e^{-i g}$, we obtain

$$
\begin{aligned}
\mathcal{L} w-g_{\tau}+\frac{1}{2 \tau^{2}} g_{\xi}^{2}= & -\frac{1}{2 \tau^{2}}\left(2 i g_{\xi} w_{\xi}+i g_{\xi \xi} w\right) \\
& +\frac{a}{\tau}\left(q|w|^{2} w+Q\right) .
\end{aligned}
$$

We now define the function $g$ as a solution of the Cauchy problem

$$
g_{\tau}=-\frac{a}{\tau} q|w|^{2}+\frac{1}{2 \tau^{2}} g_{\xi}^{2}, \tau \geq 1,
$$

with initial condition $g(1)=0$. From (2.2) it follows that

$$
\mathcal{L} w=-\frac{1}{2 \tau^{2}}\left(2 i g_{\xi} w_{\xi}+i g_{\xi \xi} w\right)+\frac{a}{\tau} Q .
$$

Thus we get the following system of equations:

$$
\left\{\begin{array}{c}
\mathcal{L} w=-\frac{1}{2 \tau^{2}}\left(2 i g_{\xi} w_{\xi}+i g_{\xi \xi} w\right)+\frac{a}{\tau} Q, \tau \geq 1, \\
g_{\tau}=-\frac{a}{\tau} q|w|^{2}+\frac{1}{2 \tau^{2}} g_{\xi}^{2}, \tau \geq 1, \\
g(1)=0, \quad w(1)=v(1)=\epsilon \sqrt{i} \mathcal{M}(1) u_{0} .
\end{array}\right.
$$

Note that via the conditions of Theorem 1.1 as in [14] we can see that the norm $\|w(1)\|_{\mathcal{H}_{\beta}^{5 / 2,3}}$ is sufficiently small. We now prove the estimates for the solution of system (2.3).

Theorem 2.1. Suppose that $|a| \leq C \tau^{1-\delta}$ and the initial data $u_{0}$ are such that the value $\varepsilon=\epsilon\left\|\mathcal{M}(1) u_{0}\right\|_{\mathcal{H}_{\beta}^{5 / 2,3}}$ is sufficiently small. Then there exists a unique solution $w \in \mathbf{C}\left([1, \infty), \mathcal{H}_{\beta / 2}^{0,3}\right), g \in \mathbf{C}\left([1, \infty), \mathcal{H}_{\beta / 2}^{2,3}\right), g_{\xi} \in \mathbf{C}\left([1, \infty), \mathcal{H}_{\beta / 2}^{1,3}\right)$ to the Cauchy problem (2.3) and the estimates

$$
\|w(\tau)\|_{\mathcal{H}_{\sigma(\tau)}^{5 / 2,3}}+\tau^{\delta-1-\gamma}\left\|g_{\xi}(\tau)\right\|_{\mathcal{H}_{\sigma(\tau)}^{5 / 2,3}}+\tau^{\delta-1}\|g(\tau)\|_{\mathcal{H}_{\beta / 2}^{2,3}} \leq C \varepsilon
$$

are valid for all $\tau \geq 1$, where $\sigma(\tau)=\beta\left(1+\tau^{-\gamma}\right) / 2,0<\gamma \leq \delta / 2$.

Proof. We consider the linearized version of system (2.3),

$$
\left\{\begin{array}{c}
\mathcal{L} w=-\frac{1}{2 \tau^{2}}\left(2 i \tilde{g}_{\xi} \tilde{w}_{\xi}+i \tilde{g}_{\xi \xi} \tilde{w}\right)+\frac{a}{\tau} \tilde{Q}, \tau \geq 1, \\
g_{\tau}=-\frac{a}{\tau} \tilde{q}|\tilde{w}|^{2}+\frac{1}{2 \tau^{2}} \tilde{g}_{\xi}^{2}, \tau \geq 1, \\
g(1)=0, \quad w(1)=\epsilon \sqrt{i} \mathcal{M}(1) u_{0},
\end{array}\right.
$$

where $\tilde{Q}=Q\left(\tilde{w}, \tilde{w}_{\xi}, \tilde{g}_{\xi}\right), \tilde{q}=q\left(\tilde{g}_{\xi}\right)$. We denote the function space

$$
\mathbf{X}=\left\{\left(\begin{array}{c}
\phi \\
\psi
\end{array}\right) ; \phi, \psi \in \mathbf{C}\left([1, \infty), \mathbf{L}^{2}\right), \quad\left\|\left(\begin{array}{c}
\phi \\
\psi
\end{array}\right)\right\|_{\mathbf{X}}<\infty\right\},
$$


where

$$
\begin{aligned}
& \left\|\left(\begin{array}{c}
\phi \\
\psi
\end{array}\right)\right\|_{\mathbf{X}}^{2} \\
& =\sup _{1 \leq \tau<\infty}\left(\|\phi(\tau)\|_{\mathcal{H}_{\sigma(\tau)}^{5 / 2,3}}^{2}+\tau^{2(\delta-1-\gamma)}\left\|\psi_{\xi}(\tau)\right\|_{\mathcal{H}_{\sigma(\tau)}^{5 / 2,3}}^{2}+\tau^{2(\delta-1)}\|\psi(\tau)\|_{\mathcal{H}_{\beta / 2}^{2,3}}^{2}\right) \\
& \quad+\int_{1}^{\infty}\left|\sigma^{\prime}(\tau)\right|\left(\|\phi(\tau)\|_{\mathcal{H}_{\sigma(\tau)}^{3,3}}^{2}+\tau^{2(\delta-1-\gamma)}\left\|\psi_{\xi}(\tau)\right\|_{\mathcal{H}_{\sigma(\tau)}^{3,3}}^{2}\right) d \tau .
\end{aligned}
$$

We denote by $\mathbf{X}_{\rho}$ the closed ball in $\mathbf{X}$ with a center at the origin and a radius $\rho>0$. We define the mapping $\mathcal{A}$ by

$$
\left(\begin{array}{l}
w \\
g
\end{array}\right)=\mathcal{A}\left(\begin{array}{c}
\tilde{w} \\
\tilde{g}
\end{array}\right) \text {, where }\left(\begin{array}{c}
\tilde{w} \\
\tilde{g}
\end{array}\right) \in \mathbf{X}_{\rho},
$$

and $w, g$ are the solutions of the Cauchy problem 2.4 . Denote $h=\tau^{\delta-1-\gamma} g_{\xi}$, $\tilde{h}=\tau^{\delta-1-\gamma} \tilde{g}_{\xi}$; then from the system (2.4) we get

$$
\left\{\begin{array}{c}
\mathcal{L} w=\tilde{G}_{1}, \tau \geq 1, w(1)=\sqrt{i} \mathcal{M}(1) u_{0}, \\
h_{\tau}=-(1+\gamma-\delta) \tau^{-1} \tilde{h}+\tilde{G}_{2}, \tau \geq 1, h(1)=0,
\end{array}\right.
$$

where

$$
\left\{\begin{array}{c}
\tilde{G}_{1}=-\frac{1}{2 \tau^{1+\delta-\gamma}}\left(2 \tilde{h} \tilde{w}_{\xi}+\tilde{w} \tilde{h}_{\xi}\right)+\frac{a}{\tau} \tilde{Q} \\
\tilde{G}_{2}=-\frac{1}{\tau^{1+\delta-\gamma}} \tilde{h} \tilde{h}_{\xi}+a \tau^{\delta-1-\gamma} \partial_{\xi}\left(\tilde{q}|\tilde{w}|^{2}\right)
\end{array}\right.
$$

Taking the Fourier transform of (2.5), multiplying the equations of the resulting system by $E^{2}(\tau, \xi)\left(\left\langle i \partial_{\xi}\right\rangle^{3} \overline{\hat{w}(\tau, \xi)}\right)\left\langle i \partial_{\xi}\right\rangle^{3}$ and $E^{2}(\tau, \xi)\left(\left\langle i \partial_{\xi}\right\rangle^{3} \overline{\hat{h}(\tau, \xi)}\right)\left\langle i \partial_{\xi}\right\rangle^{3}$ respectively, where $E(\tau, \xi)=\langle\xi\rangle^{5 / 2} e^{\sigma(\tau)|\xi|}$, integrating over $\xi \in \mathbf{R}$, taking the real part of the result and using the Schwarz inequality, we obtain

$$
\left\{\begin{array}{c}
\frac{1}{2} \frac{d}{d \tau}\|w\|_{\mathcal{H}_{\sigma}^{5 / 2,3}}^{2}-\sigma^{\prime}\left\|\sqrt{|\cdot|} E\left\langle i \partial_{\xi}\right\rangle^{3} \hat{w}\right\|^{2} \leq\|w\|_{\mathcal{H}_{\sigma}^{3,3}}\left\|\tilde{G}_{1}\right\|_{\mathcal{H}_{\sigma}^{2,3}}, \\
\frac{1}{2} \frac{d}{d \tau}\|h\|_{\mathcal{H}_{\sigma}^{5 / 2,3}}^{2}-\sigma^{\prime}\left\|\sqrt{|\cdot|} E\left\langle i \partial_{\xi}\right\rangle^{3} \hat{h}\right\|^{2}+\frac{1+\gamma-\delta}{\tau}\|h\|_{\mathcal{H}_{\sigma}^{5 / 2,3}}^{2} \leq\|h\|_{\mathcal{H}_{\sigma}^{3,3}}\left\|\tilde{G}_{2}\right\|_{\mathcal{H}_{\sigma}^{2,3}}
\end{array}\right.
$$

By Sobolev's embedding inequality we get

$$
\begin{aligned}
\left\|\tilde{h}(\tau) \tilde{w}_{\xi}(\tau)\right\|_{\mathcal{H}_{\sigma}^{2,3}} & \leq C\left(\left\|\tilde{h} \tilde{w}_{\xi}(\tau, \cdot+i \sigma)\right\|_{2,3}+\left\|\tilde{h} \tilde{w}_{\xi}(\tau, \cdot-\sigma)\right\|_{2,3}\right) \\
& \leq C \rho\|\tilde{w}(\tau)\|_{\mathcal{H}_{\sigma}^{3,3}}
\end{aligned}
$$

Similarly $\left\|\tilde{h}_{\xi} \tilde{w}\right\|_{\mathcal{H}_{\sigma}^{2,3}} \leq C \rho\|\tilde{h}\|_{\mathcal{H}_{\sigma}^{3,3}}$. Writing an analytic continuation $w(\tau, z) \overline{w(\tau, \bar{z})}$ of the nonlinearity $|w(\tau, \xi)|^{2}$ for the complex values $z$ of the independent variable $\xi$ in the strip $-\sigma \leq \Im z \leq \sigma$, we obtain

$$
\|\tilde{Q}\|_{\mathcal{H}_{\sigma}^{2,3}} \leq C \tau^{-1}\left(\rho+\rho^{5}\right)\|\tilde{w}\|_{\mathcal{H}_{\sigma}^{3,3}}
$$

therefore

$$
\left\|\tilde{G}_{1}(\tau)\right\|_{\mathcal{H}_{\sigma}^{2,3}} \leq C \rho \tau^{\gamma-1-\delta}\left(\|\tilde{w}(\tau)\|_{\mathcal{H}_{\sigma}^{3,3}}+\|\tilde{h}(\tau)\|_{\mathcal{H}_{\sigma}^{3,3}}\right)
$$

In the same manner we have

$$
\begin{aligned}
\left\|\tilde{G}_{2}(\tau)\right\|_{\mathcal{H}_{\sigma}^{2,3}} & \leq C \tau^{-\gamma-1}\left\|\left(\tilde{q}|\tilde{w}|^{2}\right)_{\xi}\right\|_{\mathcal{H}_{\sigma}^{2,3}}+C \tau^{\gamma-1-\delta}\left\|\tilde{h}_{h_{\xi}}\right\|_{\mathcal{H}_{\sigma}^{2,3}} \\
& \leq C\left(\rho+\rho^{4}\right) \tau^{-\gamma-1}\left(\|\tilde{w}(\tau)\|_{\mathcal{H}_{\sigma}^{3,3}}+\|\tilde{h}(\tau)\|_{\mathcal{H}_{\sigma}^{3,3}}\right) .
\end{aligned}
$$


Therefore denoting $J^{2}(\tau)=\|w(\tau)\|_{\mathcal{H}_{\sigma(\tau)}^{5 / 2,3}}^{2}+\|h(\tau)\|_{\mathcal{H}_{\sigma(\tau)}^{5 / 2,3}}^{2}$ we get from system (2.6)

$$
\frac{d}{d \tau} J^{2}+\left|\sigma^{\prime}(\tau)\right|\left(\|w\|_{\mathcal{H}_{\sigma}^{3,3}}^{2}+\|h\|_{\mathcal{H}_{\sigma}^{3,3}}^{2} \leq C \tau^{-1-\gamma} \rho\left(\|\tilde{w}\|_{\mathcal{H}_{\sigma}^{3,3}}+\|\tilde{h}\|_{\mathcal{H}_{\sigma}^{3,3}}\right),\right.
$$

whence integrating with respect to $\tau$, we get

$$
\begin{aligned}
J^{2}(\tau) & +\int_{1}^{\tau}\left|\sigma^{\prime}(s)\right|\left(\|w\|_{\mathcal{H}_{\sigma(s)}^{3,3}}^{2,3}+\|h\|_{\mathcal{H}_{\sigma(s)}^{3,3}}^{2,3}\right) d s \\
& \leq J^{2}(1)+C \rho \int_{1}^{\tau}\left|\sigma^{\prime}(s)\right|\left(\|\tilde{w}\|_{\mathcal{H}_{\sigma(s)}^{3,3}}^{3,3}+\|\tilde{h}\|_{\mathcal{H}_{\sigma(s)}^{3,3}}\right) d s \leq C \varepsilon^{2}+C \rho^{3} \leq C \varepsilon^{2}
\end{aligned}
$$

if the value $\rho$ is sufficiently small $C \rho^{3} \leq \varepsilon^{2}$. Again we use (2.4) to get $\|g(\tau)\|_{\mathcal{H}_{\beta / 2}^{2,3}} \leq$ $C \varepsilon^{2} \tau^{1-\delta}$. Hence we have

$$
\left\|\left(\begin{array}{c}
w \\
g
\end{array}\right)\right\|_{\mathbf{X}}=\left\|\mathcal{A}\left(\begin{array}{c}
\tilde{w} \\
\tilde{g}
\end{array}\right)\right\|_{\mathbf{X}} \leq C \varepsilon
$$

therefore $\mathcal{A}$ is a mapping from $\mathbf{X}_{C \varepsilon}$ into itself. In the same way we can show that the operator $\mathcal{A}$ is a contraction mapping from $\mathbf{X}_{C \varepsilon}$ into itself. Thus there exists a unique solution of system (2.4) in X. From (2.4) and Sobolev's embedding inequality we have the continuity in time $\|g(\tau)-g(s)\|_{\mathcal{H}_{\beta / 2}^{2,3}} \leq C \varepsilon|\tau-s|$ and $\|w(\tau)-w(s)\|_{\mathcal{H}_{\beta / 2}^{0,3}} \leq C \varepsilon|\tau-s|$. This completes the proof of Theorem 2.1.

\section{Proof of the theorems}

3.1. Proof of Theorem 1.1. In Lemma 3.1 of [14] we showed

Lemma 3.1. We assume that the nonlinear term does not depend on $\overline{u_{x}}$ and the initial data are such that $\left\|e^{\beta\langle x\rangle} u_{0}\right\|_{\mathbf{H}^{3,0}} \leq C / \epsilon$. Then for some time $T>0$ there exists a unique solution $u$ of the Cauchy problem (1.1) such that

$$
\sup _{0 \leq t \leq T}\left\|e^{\beta\langle x\rangle} \mathcal{U}(-t) u(t)\right\|_{\mathbf{H}^{3,0}}<\infty .
$$

In the same way as in the proof of Lemma 3.1, we can find that there exists a unique solution $u$ of (1.1) and a time $T \geq 1$ such that

$$
\sup _{0 \leq t \leq T}\left\|e^{\beta\langle x\rangle} \mathcal{U}(-t) u(t)\right\|_{\mathbf{H}^{3,5 / 2}} \leq C \varepsilon
$$

under the condition that the initial function is small even if the nonlinear term depends on $\overline{u_{x}}$. Hence we can assume that the norm $\|w(1)\|_{\mathcal{H}_{\beta}^{5 / 2,3}}$ of the initial data to the Cauchy problem (2.3) is sufficiently small. Therefore Theorem 2.1 implies the solution of (1.1) satisfies $\mathcal{F U}(-t) u(t) \in \mathbf{C}\left([1, \infty) ; \mathcal{H}_{\beta / 2}^{5 / 2,3}\right)$. From the definitions of $\mathcal{M}, \mathcal{D}, w, g$ it follows that

$$
u(t, x)=\mathcal{M}(t) \mathcal{D}(t) w(t) \exp (i g)=(i \tau)^{-1 / 2} w(\tau, x / \tau) \exp \left(i x^{2} / 2 \tau+i g(\tau, x / \tau)\right),
$$

where $\tau=t+1$, whence we easily get

$$
\|u(t)\|_{p} \leq C \tau^{-1 / 2}\|w(\tau, \cdot \tau)\|_{p}=C \tau^{1 / p-1 / 2}\|w\|_{p} \leq C \varepsilon t^{1 / p-1 / 2}
$$


for all $2 \leq p \leq \infty$. By virtue of Theorem 2.1 and estimate (2.7) we have

$$
\begin{aligned}
\|w(t)-w(s)\|_{\mathcal{H}_{\beta / 2}^{0,3}} \leq \int_{s}^{t}\left\|w_{\tau}(\tau)\right\|_{\mathcal{H}_{\beta / 2}^{0,3}} d \tau \leq C \int_{s}^{t}\left(\left\|g_{\xi} w_{\xi}\right\|_{\mathcal{H}_{\beta / 2}^{0,3}}+\left\|w_{\xi \xi}\right\|_{\mathcal{H}_{\beta / 2}^{0,3}}\right. \\
\left.\quad+\left\|w g_{\xi \xi}\right\|_{\mathcal{H}_{\beta / 2}^{0,3}}\right) \frac{d \tau}{\tau^{2}}+\int_{s}^{t}\|Q\|_{\mathcal{H}_{\beta / 2}^{0,3}}|a| / \tau d \tau \\
\leq C \varepsilon \int_{s}^{t} \tau^{-1-\delta} d \tau \leq C \varepsilon s^{-\delta}
\end{aligned}
$$

for all $t>s>1$, since by Theorem 2.1 we see that $\|g\|_{\infty}+\left\|g_{\xi}\right\|_{\mathcal{H}_{\beta / 2}^{1,3}} \leq C \varepsilon^{2} t^{1-\delta}$. Therefore there exists a unique limit $W_{+} \in \mathcal{H}_{\beta / 2}^{0,3}$ such that $\lim _{t \rightarrow \infty} w(t)=W_{+}$in $\mathcal{H}_{\beta / 2}^{0,3}$ and thus we get

$$
u(t, x)=(i t)^{-1 / 2} \mathcal{M}(t) W_{+}(x / t) e^{i g(t, x / t)}+O\left(\varepsilon t^{-1 / 2-\delta}\right)
$$

uniformly with respect to $x \in \mathbf{R}$ since for all $2 \leq p \leq \infty$ we have

$$
\begin{aligned}
& \left\|u(t)-(i t)^{-1 / 2} \mathcal{M}(t) W_{+}(\cdot / t) e^{-i g(t, \cdot / t)}\right\|_{p} \leq C t^{-1 / 2}\left\|w(t, \cdot / t)-W_{+}(\cdot / t)\right\|_{p} \\
& \quad \leq C t^{1 / p-1 / 2}\left\|w(t)-W_{+}\right\|_{p} \leq C \varepsilon t^{1 / p-1 / 2-\delta}
\end{aligned}
$$

For the phase $g$ we obtain

$$
\begin{aligned}
g(\tau)= & -\int_{1}^{\tau} q|w(s)|^{2} a(s-1) / s d s+\int_{1}^{\tau}\left(g_{\xi}\right)^{2} / 2 s^{2} d s \\
= & -b \int_{1}^{\tau}|w(s)|^{2} a(s-1) / s d s \\
& -\int_{1}^{\tau}(q-b)|w(s)|^{2} a(s-1) / s d s+\int_{1}^{\tau}\left(g_{\xi}\right)^{2} / 2 s^{2} d s \\
= & -b \int_{1}^{\tau}|w(s)|^{2} a(s-1) / s d s+O\left(t^{1-2 \delta}\right)
\end{aligned}
$$

uniformly with respect to $x \in \mathbf{R}$. Then we write the identity

$$
\begin{aligned}
-i b \int_{1}^{\tau} a(s-1)|w(s)|^{2} / s d s= & -i b\left|W_{+}\right|^{2} \int_{0}^{t} a(s) /(s+1) d s+\Psi(\tau) \\
& -i b\left(|w(\tau)|^{2}-\left|W_{+}\right|^{2}\right) \int_{0}^{t} a(s) /(s+1) d s,
\end{aligned}
$$

where $\Psi(\tau)=-i b \int_{1}^{\tau}\left(|w(s)|^{2}-|w(\tau)|^{2}\right) a(s-1) / s d s$. Since

$$
\left\|b\left(|w(s)|^{2}-|w(\tau)|^{2}\right)\right\|_{\infty} \leq C \varepsilon\|w(s)-w(\tau)\|_{1,0} \leq C \varepsilon^{2} \tau^{-\delta}
$$

we get

$$
-i b \int_{1}^{\tau}|w(s)|^{2} a(s) /(s+1) d s=-i b\left|W_{+}\right|^{2} \int_{0}^{t} a(s) /(s+1) d s+O\left(1+t^{1-2 \delta}\right) .
$$

From these estimates asymptotics (1.4) follows with $\hat{u}_{+}=W_{+}$. 


\subsection{Proof of Theorem 1.2. Denote}

$$
\begin{aligned}
\Phi(t)= & -b \int_{0}^{t} a(\tau)|w(\tau+1)|^{2} /(\tau+1) d \tau+b|w(t+1)|^{2} \int_{0}^{t} a(\tau) /(\tau+1) d \tau \\
& +\int_{0}^{t}\left(g_{\xi}\right)^{2} /\left(2(\tau+1)^{2}\right) d \tau
\end{aligned}
$$

Then we have

$$
\begin{aligned}
\Phi(t)-\Phi(s)= & -b \int_{s}^{t} a(\tau)\left(|w(\tau+1)|^{2}-|w(t+1)|^{2}\right) /(\tau+1) d \tau \\
& +b\left(|w(t+1)|^{2}-|w(s+1)|^{2}\right) \int_{0}^{t} a(\tau) /(\tau+1) d \tau \\
& +\int_{s}^{t}\left(g_{\xi}\right)^{2} /\left(2(\tau+1)^{2}\right) d \tau
\end{aligned}
$$

where $1<s<t$. We apply Theorem 2.1 and equations (3.1) to (3.3) to get $\|\Phi(t)-\Phi(s)\|_{\mathcal{H}_{\beta / 2}^{0,3}} \leq C \varepsilon s^{1-2 \delta}$ for all $1<s<t$. This implies that there exists a unique limit $\Phi_{+}=\lim _{t \rightarrow \infty} \Phi(t) \in \mathcal{H}_{\beta / 2}^{0,3}$ such that

$$
\left\|\Phi(t)-\Phi_{+}\right\|_{\mathcal{H}_{\beta / 2}^{0,3}} \leq C \varepsilon t^{1-2 \delta}
$$

since $\frac{1}{2}<\delta<1$. Furthermore

$$
\begin{aligned}
\Phi(t)= & -g(t)-b|w(t+1)|^{2} \int_{0}^{t} a(\tau) /(\tau+1) d \tau \\
& +\int_{0}^{t}(q-b)|w(s+1)|^{2} a(s) /(s+1) d s
\end{aligned}
$$

so we have by virtue of (3.2), (3.4) and Theorem 2.1

$$
\left\|g(t)+b\left|W_{+}\right|^{2} \int_{0}^{t} a(\tau) /(\tau+1) d \tau-\Phi_{+}\right\|_{\infty} \leq C \varepsilon t^{1-2 \delta}
$$

We now put $\hat{u}_{+}=W_{+} \exp \left(-i \Phi_{+}\right) \in \mathcal{H}_{\beta / 2}^{0,3}$. Therefore we obtain the asymptotics (1.5). Via (3.5) and Theorem 2.1 we have

$$
\begin{aligned}
& \left\|\mathcal{F} \mathcal{M U}(-t) u(t)-\hat{u}_{+} \exp \left(-i b\left|\hat{u}_{+}\right|^{2} \int_{0}^{t} a(\tau) /(\tau+1) d \tau\right)\right\| \\
& \quad=\left\|w(t) \exp (i g(t))-W_{+} \exp \left(-i b\left|W_{+}\right|^{2} \int_{0}^{t} a(\tau) /(\tau+1) d \tau+i \Phi_{+}\right)\right\| \\
& \quad \leq\left\|w(t)-W_{+}\right\|+\left\|W_{+}\right\|\left\|g(t)+b\left|W_{+}\right|^{2} \int_{0}^{t} a(\tau) /(\tau+1) d \tau-\Phi\right\|_{\infty} \leq C \varepsilon t^{1-2 \delta}
\end{aligned}
$$


whence we get

$$
\begin{aligned}
\left\|u(t)-\exp \left(-i b(\cdot / t)\left|\hat{u}_{+}(\cdot / t)\right|^{2} \int_{0}^{t} a(\tau) /(\tau+1) d \tau\right) \mathcal{U}(t) u_{+}\right\| \\
=\left\|u(t)-\mathcal{M}(t) \mathcal{D}(t) \exp \left(-i b\left|\hat{u}_{+}\right|^{2} \int_{0}^{t} a(\tau) /(\tau+1) d \tau\right) \mathcal{F} \mathcal{M}(t) u_{+}\right\| \\
\leq\left\|\mathcal{M}(t) \mathcal{D}(t)\left(\mathcal{F} \mathcal{M}(t) \mathcal{U}(-t) u(t)-\hat{u}_{+} \exp \left(-i b\left|\hat{u}_{+}\right|^{2} \int_{0}^{t} a(\tau) /(\tau+1) d \tau\right)\right)\right\| \\
\quad+\left\|\mathcal{M}(t) \mathcal{D}(t) \exp \left(-i b\left|\hat{u}_{+}\right|^{2} \int_{0}^{t} a(\tau) /(\tau+1) d \tau\right) \mathcal{F}(\mathcal{M}(t)-1) u_{+}\right\| \\
\leq C t^{1-2 \delta}+C\left\|\mathcal{F}(\mathcal{M}(t)-1) u_{+}\right\| \leq C t^{1-2 \delta}+C t^{-1}\left\|x^{2} u_{+}\right\| \leq C t^{1-2 \delta}
\end{aligned}
$$

since $\left\|x^{2} u_{+}\right\|=\left\|\partial_{x}^{2}\left(W_{+} e^{-i \Phi_{+}}\right)\right\| \leq C \varepsilon$. This completes the proof of Theorem 1.2.

\section{ACKNOWLEDGMEnTs}

The authors would like to thank the referee for useful comments and suggestions.

\section{REFERENCES}

1. P. D'Ancona and S. Spagnolo, Global solvability for the degenerate Kirchhoff equation with real analytic data, Invent. Math., 108 (1992), pp. 247-262. MR 93h:35131

2. J. Ginibre and T. Ozawa, Long range scattering for nonlinear Schrödinger and Hartree equations in space dimension $n \geq 2$, Commun. Math. Phys., 151 (1993), pp. 619-645. MR 93m:35168

3. J. Ginibre and G. Velo, On a class of nonlinear Schrödinger equations. I. The Cauchy problem, general case; II Scattering theory, general case, J. Funct. Anal., 32 (1979), pp. 1-71. MR 82c:35058

4. J. Ginibre and G. Velo, Scattering theory in the energy space of a class of nonlinear Schrödinger equations, J. Math. Pures Appl., 64 (1985), pp. 363-401. MR 87i:35171

5. J. Ginibre and G. Velo, Long range scattering and modified wave operators for some Hartree type equations I, Rev. Math. Phys., 12 (2000), pp. 361-429. CMP 2000:12

6. J. Ginibre and G. Velo, Long range scattering and modified wave operators for some Hartree type equations II, Ann. Inst. H. Poincaré, 1 (2000), pp. 753-800. CMP 2001:02

7. J. Ginibre and G. Velo, Long range scattering and modified wave operators for some Hartree type equations III, Gevrey spaces and low dimensions, preprint (2000).

8. N. Hayashi, Global existence of small analytic solutions to nonlinear Schrödinger equations, Duke Math. J., 60 (1990), pp. 717-727. MR 92d:35270

9. N. Hayashi, E.I. Kaikina and P.I. Naumkin, On the scattering theory for the cubic nonlinear Schrödinger and Hartree type equations in one space dimension, Hokkaido Math. J., 27 (1998), pp. 651-667. MR 99m:35228

10. N. Hayashi, K. Kato and P.I. Naumkin, On the scattering in Gevrey classes for the subcritical Hartree and Schrödinger equations, Ann. Scuol. Norm. Sup. Pisa, 27 (1998), pp. 483-497. MR 2000a:35225

11. N. Hayashi and P.I. Naumkin, Asymptotic behavior in time of solutions to nonlinear Schrödinger and the Hartree equations, Amer. J. Math., 120 (1998), pp. 369-389. MR 99f:35190

12. N. Hayashi and P.I. Naumkin, Scattering theory and large time asymptotics of solutions to the Hartree type equations with a long range potential, Hokkaido Math. J., 30 (2001), 137-161. CMP 2001:08

13. N. Hayashi and P.I. Naumkin, Large time behavior of solutions for derivative cubic nonlinear Schrödinger equations without a self-conjugate property, Funk. Ekva., 42 (1999), pp. 311-324. MR 2000h:35149

14. N. Hayashi, P.I. Naumkin and P.N. Pipolo, Analytic smoothing effects for some derivative nonlinear Schrödinger equations, Tsukuba J. Math., 24 (2000), 21-34. CMP 2001:03 
15. N. Hayashi, P.I. Naumkin and H. Uchida, Large time behavior of solutions for derivative cubic nonlinear Schrödinger equations, Publ. RIMS Kyoto Univ., 35, No. 3 (1999), 501-513. MR 2000j:35246

16. N. Hayashi and T. Ozawa, Scattering theory in the weighted $\mathbf{L}^{2}\left(\mathbf{R}^{n}\right)$ spaces for some Schrödinger equations, Ann. Inst. H. Poincaré (Phys. Théor.), 48 (1988), pp. 17-37. MR 89k:35175

17. N. Hayashi and Y. Tsutsumi, Remarks on the scattering problem for nonlinear Schrödinger equations, Diff. Equs. and Math. Phys., Lect. Note in Math., Edited by I.W. Knowles and Y. Saito, Springer-Verlag, 1285 (1986), pp. 162-168. MR 89d:35155

18. T. Kato and K. Masuda, Nonlinear evolution equations and analyticity I, Ann. I.H.P. (Anal. non Linéaire), 3 (1986), pp. 455-467. MR 88h:34041

19. S. Klainerman and G. Ponce, Global, small amplitude solutions to nonlinear evolution equations, Commun. Pure Appl. Math., 36 (1983), pp. 133-141. MR 84a:35173

20. P.I. Naumkin, Asymptotics for large time for nonlinear Schrödinger equation, Izvestiya: Math., 61, No. 4 (1997), pp. 757-794. MR 98j:35171

21. T. Ozawa, Long range scattering for nonlinear Schrödinger equations in one space dimension, Commun. Math. Phys., 139 (1991), pp. 479-493. MR 92j:35172

22. T. Ozawa, Remarks on the quadratic nonlinear Schrödinger equations, Funkc. Ekva., 38, No. 2 (1995), pp. 217-232. MR 96j:35238

23. J. Shatah, Global existence of small solutions to nonlinear evolution equations, J. Diff. Equs., 46 (1982), pp. 409-425. MR 84g:35036

24. J.C.H. Simon and E. Taflin, Wave operators and analytic solutions of non-linear KleinGordon equations and of non-linear Schrödinger equations, Commun. Math. Phys., 99 (1985), pp. 541-562. MR 86j:35147

25. Y. Tsutsumi, Scattering problem for nonlinear Schrödinger equations, Ann. I.H.P. (Phys. Théor.), 43 (1985), pp. 321-347. MR 87g:35221

Department of Applied Mathematics, Science University of Tokyo, Tokyo 162-8601, JAPAN

E-mail address: nhayashi@rs.kagu.sut.ac.jp

Current address: Department of Mathematics, Graduate School of Science, Osaka University,

Toyonaka, Osaka 560-0043, Japan

E-mail address: nhayashi@math.wani.osaka-u.ac.jp

Instituto de Física y Matemáticas, Universidad Michoacana, AP 2-82, CP 58040, Morelia, Michoacán, México

E-mail address: pavelni@zeus.ccu.umich.mx

Department of Applied Mathematics, Science University of Tokyo, Tokyo 162-8601, JAPAN

Current address: Department of Mathematics, Graduate School of Science, Hokkaido University, Sapporo 060, Japan

E-mail address: yamazaki@math.sci.hokudai.ac.jp 